\title{
El análisis histórico, epistemológico y didáctico como una concreción del modelo de aprendizaje de la termodinámica por investigación
}

\author{
Yair Alexander Porras Contreras*
}

Artículo recibido: 20-02-2006 y aprobado: 28-09-2006

The historic, epistemological and didactic analysis as a concreteness of the learning model thermodynamic like an oriented research

Resumen: Con este artículo se pretende generar una propuesta didáctica en la que se enfatice la evolución conceptual de los estudiantes con base en un estudio de los aspectos históricos y epistemológicos, una secuenciación de contenidos que tenga presentes las dificultades en la construcción de conceptos y una opción de trabajo en el aula que permita transformar la enseñanza habitual de los conceptos termodinámicos.

Palabras clave: Historia, epistemología, didáctica, resolución de problemas, calor, temperatura, energía.

Key words: History, epistemology, didactics, problem solving, heat, temperature, energy. present article consists on generating a didactic proposal in which the conceptual evolution of the students is emphasized with base in a study of the historical and epistemological aspects, a sequence of contents that has present the difficulties in the construction of concepts and a working alternative in the classroom that allows the transformation of the habitual teaching of the thermodynamic concepts, in which a bigger interest is evidenced toward the learning of the science.

\footnotetext{
Profesor de la Universidad Pedagógica Nacional. yairporras@yahoo.es
}

$\mathbf{T \in \Delta}$ N. $^{\circ} 20 \cdot$ Segundo semestre de $2006 \cdot$ pp. 17-41 $17 \mid \mathrm{T} \in \Delta$ 


\section{Introducción}

Realizar un análisis histórico y epistemológico sobre la construcción y desarrollo de los conceptos energía interna, calor y temperatura, sin desconocer la conexión existente con otros conceptos afines como el trabajo, la energía libre, la entalpía, la entropía, etc., permite evidenciar la estructuración de una serie de esquemas de interpretación del mundo, en los cuales se fundamenta un conjunto de acuerdos centrales que organizan la discusión frente a la definición de los problemas estructurales de las disciplinas, las estrategias que se van a utilizar y la formulación de opciones para solucionar a dichas problemáticas, reconociendo a su vez el contexto en el cual se cimientan las comunidades académicas y la influencia del clima social de la época en que se construye el conocimiento.

Tales acuerdos centrales son lo que Kuhn (1992) denomina paradigmas, aquellas realizaciones que una comunidad científica reconoce, los cuales involucran teorías, leyes, aplicaciones, instrumentos y técnicas, por lo que se analiza este término en relación con los esquemas de comprensión de la realidad que la comunidad científica maneja, y que influyen en la actuación profesional. Un paradigma que involucra los supuestos teóricos generales de una disciplina y sus técnicas de aplicación, avalados por una comunidad científica, se halla inmerso en la denominada ciencia normal, es decir, aquella investigación basada firmemente en una o varias realizaciones pasadas, realizaciones que alguna comunidad científica particular reconoce durante cierto tiempo como fundamento para su práctica posterior (Kuhn, 1992), lo que en esencia permite concluir que la labor de dicha comunidad científica se traduce en una investigación dominada por paradigmas. Esta posición frente a la realidad nos sugiere que la ciencia se constituye en una labor de comprensión del mundo, en la que los sujetos y el colectivo construyen modelos a partir de determinados paradigmas, los cuales varían a lo largo del tiempo al reconocerse nuevas regularidades en hechos $u$ objetos, y reconstruyendo, ampliando o reestructurando las ideas propias, para llegar a nuevas relaciones de orden superior (Novak, 1988).

Somos protagonistas de un periodo de cambios en relación con el sentido de la enseñanza y el aprendizaje, pero también somos testigos de la aceptación de ciertas posturas que propugnan la verdad de las teorías, la utilización del método científico sin excepción y el descubrimiento de las leyes naturales. Si bien es cierto que en el debate epistemológico contemporáneo estas posiciones se han superado en gran parte (Pozo, 1998), no puede decirse lo mismo en el ámbito de las instituciones educativas por cuanto prevalece la idea de que las teorías científicas son saberes absolutos, que hacen uso de un método adecuado en el que se sobredimensionan tanto las observaciones como las generalizaciones acríticas y se deja de lado un enfoque dialéctico sobre el cual puede formarse un verdadero espíritu científico (Bachelard, 1983).

\section{Revisión histórica de la ley de conservación de la energía}

Uno de los conceptos más prolíficos e integradores, que denota la preocupación creciente de la humanidad por 
unificar las leyes que rigen el universo, es sin duda alguna la energía. El análisis histórico en torno a este concepto nos revela que sus orígenes datan de la ontología aristotélica (384-322 a.C.), en la que el pensamiento materialista supone que los sentidos son parte fundamental del proceso de conocimiento, proponiéndose una metodología inductiva para el entendimiento de la energía, que se enmarca en dos rasgos de los sucesos: el movimiento de los cuerpos en el espacio y la naturaleza del calor.

En este sentido, las explicaciones a los fenómenos observados se centraban en ejemplos utilizados por Aristóteles, en los que se opinaba sobre cómo debería ser el movimiento de objetos como flechas y piedras, declarando la necesidad de una fuerza de apoyo, lo que hacía pensar en esta época que una flecha se mantenía en pleno vuelo gracias a torbellinos y vórtices que formaba el aire (Atkins, 2002). Por otra parte, al considerarse el fuego uno de los cuatro elementos de la antigüedad, el comportamiento de los cuerpos calientes se explicaba de manera cualitativa, obedeciendo ciertas leyes de la naturaleza en las que la materia buscaba su sitio natural. Recordemos que la cosmovisión aristotélica concebía el universo como una serie de esferas concéntricas en las que se encontraba la Tierra, rodeada de la esfera agua, la esfera aire y la esfera fuego; por tanto, el movimiento de una llama se explicaba por su tendencia natural de ubicarse en la última esfera; quizás ésta sea la explicación sobre el uso de

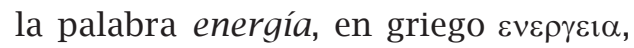
cuya traducción significa "en marcha" (Bensaude-Vincent, 1997).
Los mismos atomistas griegos aportaron explicaciones novedosas en torno a las diferencias de temperatura de los cuerpos, concibiendo el calor como una sustancia atómica especial, que se difundía a través de los cuerpos.

Poco a poco la imagen se hizo más precisa: se trataba de un fluido tenue, capaz de entrar y salir a través de los "poros" más pequeños, cuya magnitud dependía de la temperatura; era imponderable y, quizás, semejante al fluido sutil y omnipresente de Descartes o los éteres invocados por alguien para explicar la gravitación, la propagación de la luz y del calor radiante, la transmisión de las fuerzas eléctricas y magnéticas, etc. (Holton, 1986).

La influencia de estas ideas prevaleció por siglos, ocupando un sitial de honor al constituirse en verdades casi irrefutables, por lo que la crisis de esta interpretación de los acontecimientos llegó en el siglo XVII, junto con los cambios sociales en la burguesía. Uno de los intentos por comprender las verdades que se ocultaban en la naturaleza, en contraposición al Organum aristotélico, lo realizó Francis Bacon (1980), quien propugnó el acercamiento a la verdad de los fenómenos apoyado en una metodología empiro-inductivista, en la que el mundo es externo al sujeto que conoce, y el acceso al conocimiento debe entenderse como la incorporación de la realidad, tomando fiel copia de sus detalles y acumulando una serie de datos discriminados en tres tablas (presencias, ausencias y relaciones), que verificarían la fidelidad de tal impronta sólo si el sujeto está "libre" de prejuicios. De acuerdo con esta metodología era probable desconfiar de la teoría del 
calor como un fluido, para aceptar, en principio, una explicación en la que se concebía el calor como una vibración de las partículas de los cuerpos calientes, tal como lo mostraba la experiencia al provocar la fricción entre dos materiales.

El calor es un movimiento expansivo, porque el cuerpo lucha por dilatarse, y habitar un espacio más grande. Esta diferencia es vista principalmente en la llama, donde el humo o el vapor grueso están evidentemente dilatados cuando se ponen a la llama (...) el calor no es una señal expansiva y uniforme del todo, sino de las partículas pequeñas del cuerpo (...) Pero esta diferencia es mostrada principalmente en la llama y los líquidos hirviendo, que se apuran, se hinchan, y se hunden otra vez siempre en partes separadas... (Bacon, 1620).

Sin lugar a dudas, el siglo XVII marcó el inicio de transformaciones en el pensamiento de la humanidad, que se condensaron en la mentalidad abierta del Renacimiento a través del trabajo de personajes como Galileo y Newton, quienes aplicaron los principios matemáticos, heredados del pensamiento antiguo, en la geometrización de la realidad, asignando un carácter de contrastación matematizable e instrumental a sus teorías. De hecho uno de ellos, Galileo, se circunscribe en la historia como uno de los primeros en corroborar un principio teórico predecible, mediante el trabajo experimental. Así, su crítica a las explicaciones aristotélicas en relación con el movimiento se centró en poner de relieve la abstracción al establecer que un cuerpo mantenía su estado de movimiento sin que hubiera una fuerza que lo empujara.
Retomando estas ideas de Galileo y profundizando en la relación de las matemáticas y la física, Isaac Newton continuó la obra de Euclides al describir el movimiento en ese espacio idealizado desde la geometría. Sus tres leyes del movimiento sirvieron más adelante para inspirar la forma de comprender la inercia de un cuerpo (primera ley), predecir la trayectoria de una partícula que esté sujeta a una fuerza dada (segunda ley) $\mathrm{y}$, como parte fundamental de la historia de la energía, la existencia de una ley de conservación (tercera ley), traducida en la conservación del impulso lineal.

\section{El concepto de vis viva y su relación con la conservación de la energía}

Un contemporáneo de Newton, llamado por él mismo uno de los tres mayores geómetras de su tiempo, fue el físico holandés Christian Huygens (1629-1695), quien propuso en 1669 que en el caso especial de choques perfectamente elásticos, además de la cantidad de movimiento, se conservaba el producto de la masa por la velocidad al cuadrado $\left(m v^{2}\right)$, término denominado más tarde vis viva o "fuerza viva" (Holton, 1986), quizás para denotar el vigor de los sucesos que ocurren en la interacción de una serie de partículas. De hecho, 138 años después, el primer pensador que empleó el término energía desde una perspectiva científica, Thomas Young (1773-1829), escribió: "El término energía puede aplicarse, con gran propiedad, al producto de la masa o peso de un cuerpo por el cuadrado del número que expresa su velocidad" (Atkins, 2002), lo que exaltó el trabajo de Huygens, aunque la comunidad académica de la época no tardó mucho en encontrar anomalías a dicha 
aseveración. Según Young, la fuerza que ejerce un objeto cuando choca contra otro podría expresarse como vis viva además, dicha fuerza podría aumentar cuatro veces si se duplicara su velocidad. Esta forma ingeniosa de concebir los choques tenía irregularidades en el factor numérico, que sólo se reconocieron en 1820 cuando se relacionó el concepto trabajo con la segunda ley de Newton: Trabajo $=$ Fuerza $*$ desplazamiento $=1 / 2$ (vis viva).

Aquí, la energía procedente del movimiento se expresa como la mitad de su cantidad. El trabajo se realiza siempre que un objeto se mueve contra una fuerza opuesta, lo que puede ejemplificarse cuando movemos un bloque sobre una superficie que ofrece una fricción mínima, como una pista de hielo. Si lo empujamos, acelera y su energía cinética aumenta, hasta que siga resbalando y alcance una velocidad constante; en este momento, el trabajo realizado se puede asociar a la energía cinética o energía del movimiento, con lo cual es pertinente recordar la segunda ley de Newton: "La aceleración del cuerpo es proporcional a la fuerza aplicada". Si vamos más allá en el análisis, podríamos afirmar que la confusión entre energía y trabajo radica particularmente en que este último se asocia al trabajo externo, por lo que analogías en las que se pone de manifiesto el concepto de energía como la capacidad de realizar un trabajo, surgen del teorema del trabajo y la energía cinética aplicada a una partícula (Michinel y D’Alessandro, 1994).

Igualmente la energía potencial, definida como aquella que se posee en virtud de una ubicación, la concibió Rankine, en 1853, como una capacidad para realizar trabajo, por lo que consideró dicha forma de energía se consideró propicia para convertirse en vis viva o energía cinética. Esta afirmación revela un punto crucial en la historia de la ciencia, pues se habló del comportamiento de las partículas dentro de una energía total, como resultado de la suma de las energías cinética y potencial, con lo que se llegó así, en forma inicial, a la ley de conservación de la energía, consignada en el libro Tratado de filosofía natural, de Thomson y Tait, en 1867 (BensaudeVincent, 1997). Pese a este avance en el pensamiento científico, existía un riesgo latente que amenazaba con echar al piso todos los esfuerzos; este desafío convergía en el reconocimiento de la naturaleza del calor, un concepto bastante complejo dentro del entramado de ideas que se iban tejiendo paulatinamente en esta época.

\section{La teoría del calórico}

Como vemos, después de un recorrido general por los acontecimientos e historias de vida de aquellos científicos preocupados por las propiedades de la energía, la naturaleza del calor recobró un sitial importante en la discusión académica del siglo XVIII, por cuanto el estudio de sus características no era compatible con los alcances de la física de la época y existía una creencia generalizada de asociarlo con un fluido denominado calórico (proveniente del latín), que poseía la peculiaridad de ser "imponderable" y "sutil", por cuanto podría penetrar en los cuerpos.

Uno de los defensores de la teoría del calórico fue Joseph Black (1728-1799), quien concluyó, con experimentos en los cuales se ponían en contacto dos cuer- 
pos a diferente temperatura, que el calor no se creaba ni se destruía, sólo podía transferirse de un cuerpo a otro.

Comenté antes que, incluso sin la ayuda de termómetros, podemos percibir una tendencia del calor a difundirse de cualquier cuerpo más caliente a todas las partes de una heladera, hasta que es distribuido, de tal manera que ninguno de ellos está dispuesto a tomar más calor que el resto. El calor es causado por un estado de equilibrio. Este equilibrio es algo extraño(...) Debemos adoptar, por tanto, como una de las leyes más generales del calor, que: Todos los cuerpos se comunican entre sí libremente, y sin exponerse a la acción externa, adquieren la misma temperatura, como se demuestra con un termómetro. Todos adquieren la temperatura del medio circundante'” (Black, 1803).

Por ejemplo, si un bloque caliente (A) se coloca en una cantidad de agua fría (B), según Black, el fluido calorífico fluye del cuerpo más caliente al frío y alcanza así su equilibrio, lo que podría simbolizarse como $\triangle \mathrm{QA}+\Delta \mathrm{QB}=0$

$\Delta \mathrm{Q}$ es la variación de calor de cualquier muestra, que puede calcularse a partir de la siguiente ecuación:

$\Delta \mathrm{Q}=$ masa de la muestra $(\mathrm{g}) \mathrm{X}$ calor específico (cal/g $\left.{ }^{\circ} \mathrm{C}\right) \mathrm{X}$ (temp. final $\left({ }^{\circ} \mathrm{C}\right)$ - temp. inc. $\left.\left({ }^{\circ} \mathrm{C}\right)\right)$

Como se puede inferir de la ecuación anterior, no era de extrañarse que los estudiosos de la época asociaran el calor con una sustancia material, pues era fácil pensar que "el exceso de 'fluido calorífico’ fluye del cuerpo más caliente al más frío, hasta alcanzar el equilibrio. $\mathrm{Y}$, realmente, era casi inconcebible que tan simple observación exigiera el punto de vista del calor como un modo de movimiento de las partículas" (Bartell, 2001). Uno de los experimentos de Black consistió en mezclar mercurio a $150^{\circ}$ con agua a $100^{\circ}$, resultando una temperatura final de sólo $120^{\circ}$, con lo que pensó que el mercurio se tornaba $20^{\circ}$ menos caliente, mientras que el agua se hacía $20^{\circ}$ más caliente, concluyendo que cada sustancia captaba una cantidad propia de calor, que llamó calor específico (Leicester, 1967).

Se pensaba que el calórico estaba formado por partículas minúsculas que se repelían entre sí, pero eran atraídas por los corpúsculos de la materia. Como Black había sido un precursor de los experimentos con gases (en su libro Experiments upon magnesia alba, quicklime, and some other alcaline substances demostró que un gas podía combinarse -fijarse-químicamente con un sólido), imaginó que las partículas del calórico formaban una atmósfera alrededor de los corpúsculos de la materia y, de acuerdo con el estado físico del material, tenderían a dispersarse, lo que en esencia explicaría la dilatación de los cuerpos al repelerse las partículas del calórico, sobre todo en líquidos y sólidos.

Así, en cambios bruscos de estado las partículas de calórico se reacomodarían, trasladándose parte del calórico a otros cuerpos, por lo que cambiaría de un estado "latente" a otro "sensible" (Bensaude-Vincent, 1997). Cuestiones mecánicas como las torsiones de sólidos, la compresión de un gas, algunas reacciones químicas, etc., explicarían la salida del calórico por reducción de los espacios entre las partículas. El desarrollo de esta teoría tuvo su punto máximo de aceptación en la década de 1780 a 1790, pero no tardó mucho en re- 
batirse, en cierta forma, al comprobarse la imposibilidad de pesar el calórico en 1798, gracias a los trabajos del científico norteamericano Benjamin Thompson, conde de Rumford' (1753 - 1814), quien huyó a Inglaterra durante la guerra de independencia, y llegó a ser caballero y ministro de guerra en la corte del rey Jorge III.

Precisamente en las labores propias de este último cargo, al revisar la fabricación de cañones, notó que el taladro adquiría un grado de calor tan alto que podía hacer que hirviera el agua con la que se evitaba el calentamiento extremo, concluyendo que el calor se producía no por el desgaste de la herramienta, sino por un movimiento vibratorio de las partículas (a pesar de que el taladro estuviese desgastado), de manera que se podía obtener calor en forma continua e inagotable. Así, en una crítica al calórico advierte:

¿Qué es el calor? ¿Hay alguna cosa llamada fluido ígneo? ¿Hay algo que puede llamarse con decoro calórico? Hemos visto que una cantidad muy considerable del calor puede ser excitada en el rozamiento de dos superficies metálicas y dada en un torrente continuo o cambio continuo, en todas direcciones, sin la repetición o el intermedio, y sin alguna señal de disminución o agotamiento(...) ¿Fue suministrado por el agua que rodeó la maquinaria? Esto no pudo haber sido: primero, porque el agua estaba recibiendo el calor de la maquinaria continuamente y no podía estar dan-

1 Este polifacético hombre de ciencia escogió su título en homenaje al lugar de nacimiento de su primera esposa (Rumford, en Nueva Hampshire), aunque más tarde tendría un matrimonio polémico con madame Lavoisier, esposa de Antoine, decapitado en la Revolución francesa. do calor al cuerpo y recibiendo calor del mismo cuerpo, simultáneamente y en segundo lugar, porque no había descomposición química de ninguna parte de esta agua (Thompson, 1798).

Estos planteamientos desacreditaron, en alguna medida, las ideas de Black al negar la posibilidad de un principio de conservación del calor, por lo que no tardaron muchos en reformular de las explicaciones planteadas desde la teoría del calórico. Paradójicamente, el mismo cuerpo teórico que mostró las flaquezas de las conclusiones de Rumford, el calor radiante, que consideraba el calor una sustancia diferente que viajaba en el espacio, acrecentó la visión corpuscular del mismo, al asociarse las propiedades de la luz con las propiedades del calor, significado que contribuyó a reconocerlo, desde una perspectiva newtoniana, como una sustancia compuesta por partículas. Más adelante en 1825, con los experimentos de Young y Fresnel sobre la teoría ondulatoria de la luz, se concibió el calor como un movimiento vibratorio.

\section{Equivalente mecánico del calor y la conservación de la energía}

El siglo XIX se caracterizó por los estudios orientados a medir el "equivalente mecánico del calor”, que en otras palabras significaba tener una medida del trabajo que podía obtenerse a partir de cierta cantidad de calor. Uno de los primeros en tratar este tema fue Julius Robert Mayer (1814 - 1878), quien en su "Ensayo sobre las energías" describió la conservación y equivalencia de las formas de energía, dejando un interrogante clave para deducir la equivalencia entre calor y trabajo: “¿Qué cantidad de calor 
corresponde a una cantidad dada de energía cinética y potencial?".

La respuesta se desarrolló utilizando la unidad de calor o caloría ${ }^{2}$ y el calor específico ${ }^{3}$, convenidos con anterioridad y aplicados al experimento de expansión libre del aire de Gay-Lussac, en un cilindro con un pistón que se desliza libremente. Según la teoría del calórico, la temperatura de un gas que se expande en un recipiente desciende porque el calórico se distribuye de modo uniforme en un volumen mayor, permaneciendo invariable; por el contrario, Mayer supuso que la temperatura sólo disminuye si el gas produce trabajo mecánico, por lo que el calórico desaparece. Así, llegó a la conclusión de que el calor y el trabajo eran semejantes, por lo que 1 caloría equivaldría aproximadamente a 3,6 joules.

Como es común en algunos apartados de la ciencia, es necesario demostrar desde múltiples perspectivas la crisis del paradigma vigente, por lo cual se tardó mucho en acoger las ideas de Mayer, más por su estilo particular de expresar sus argumentos ante auditorios que añoraban las explicaciones newtonianas, que por la profundidad y utilidad de sus pensamientos. En 1843 un hombre de Manchester, alumno de John Dalton, de nombre James Prescott Joule (18181889), dio a conocer una de las obras

2 La caloría se define como la cantidad de calor necesaria para elevar la temperatura en la escala Celsius de 1 gramo de agua. Para trabajos exactos, deben especificarse las temperaturas inicial y final; por ejemplo: la caloría es la cantidad de calor necesaria para elevar la temperatura de un gramo de agua de $15^{\circ}$ a $16^{\circ} \mathrm{C}$

3 El calor específico es la energía calorífica necesaria para elevar en $1^{\circ} \mathrm{C}$ la temperatura de una sustancia. claves que ocuparían casi toda su vida: la conversión del trabajo mecánico en calor. En su célebre experimento utilizó una rueda de paletas que giraba dentro de un recipiente aislado, gracias a la caída de una pesa. El trabajo realizado podía calcularse por la distancia que recorre el peso en su caída, observó la temperatura del agua y consecuentemente calculó el calor necesario para lograr el mismo efecto (figura 1).

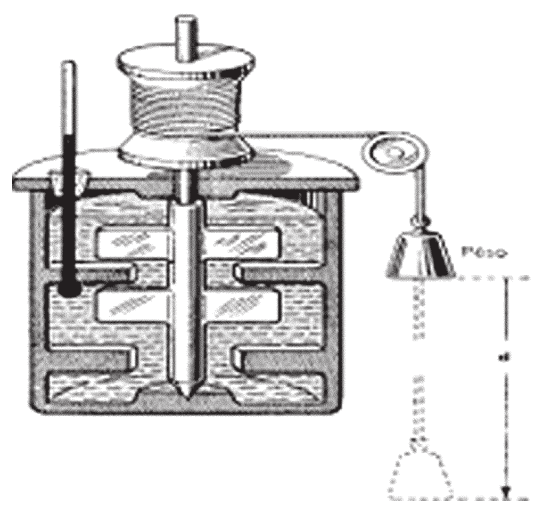

Figura 1. Experimento de Joule sobre la conversión del trabajo mecánico en calor.

Los aparatos utilizados, que se expusieron antes a la Asociación, constaban de un remo - rueda de latón trabajando horizontalmente en una lata de agua. El movimiento podía comunicarse a este remo por pesas y poleas, de la manera descrita en un trabajo previo. El remo se movió con gran resistencia en la lata del agua, y los pesos (cada uno de cuatro libras) descendieron de manera lenta, a una tasa de aproximadamente un pie por segundo. La altura de las poleas al suelo era de doce yardas, y por consiguiente, cuando los pesos habían descendido a través de esa distancia, tuvieron que ser puestos otra vez para reanudar el movimiento del remo. Después de que 
esta operación se había repetido 16 veces, el aumento de la temperatura del agua fue determinado con un termómetro muy sensible y preciso (Joule, 1845).

Al comunicar los resultados de numerosos experimentos a la Asociación Británica de Oxford (recordemos que una de las premisas del método inductivo consiste en aunar la mayor cantidad de evidencias para generar una ley), en los cuales se demostraba la conservación de la energía, se le pidió que hiciera una breve descripción del mismo; debido al poco interés que generaba, "esto intenté hacer”, escribió posteriormente, "y, sin que hubiese comunicación alguna, la información pudo haber pasado sin comentario, si un hombre joven no se hubiese levantado y creado, por sus inteligentes observaciones, un avivado interés sobre la nueva teoría. Este joven era William Thomson, lord Kelvin" (Bartell, 2001), quien diferenció implícita y tempranamente la temperatura del calor:

Definición de la temperatura y la suposición termométrica general. Si dos cuerpos son puestos en contacto, y ninguno da calor al otro, sus temperaturas deben ser las m000ismas; pero si uno da calor a los demás, su temperatura debe ser más alta. (Thomsom, -Lord Kelvin-, 1852).

Posteriormente, Joule resumió las conclusiones de todos sus trabajos, asignándole un valor de 4,15 J a una caloría, muy cerca del valor aceptado hoy, de 4,184 joules / calorías; en homenaje a su contribución a la ciencia, la unidad de medida del trabajo y el calor es el joule.

La primera redacción clara y profunda del principio general de la conser- vación de la energía la realizó Herman von Helmholtz en 1847. Este alemán, estudioso de la fisiología y la física, demostró el principio de conservación en distintos campos, entre los que se encuentran la mecánica, la electricidad, el magnetismo, la fisicoquímica y la astronomía (Bartell, 2001).

\section{Los conceptos energía interna, trabajo, calor y temperatura, a la luz de los paradigmas vigentes}

Como se puede interpretar del análisis histórico de algunos conceptos termodinámicos, resulta inapropiado hablar del calor y el trabajo como formas de energía, al igual que del equivalente mecánico del calor, de acuerdo con los paradigmas actuales en termodinámica, ya que en el experimento de Joule el trabajo no se convierte en calor sino que produce una variación en la energía interna.

Los términos calor absorbido, calor cedido, calor suministrado y calor sustraído son inapropiados por cuanto el calor no está contenido en los cuerpos, es decir, no es una función de estado; de hecho, el empleo de un lenguaje en el que se hable de aumento o disminución de la energía debe ser objeto de análisis en favor de una comprensión más consistente con los principios termodinámicos. A continuación se deduce el principio de conservación de la energía, comenzando con un análisis de los conceptos energía, calor y temperatura, como puntos de partida en la consolidación de un lenguaje científico que permita establecer una relación entre la ley general de conservación de la energía y la teoría cinético-molecular. Es conveniente recalcar el papel fundamen- 
tal de los conceptos aquí estudiados y que forman parte de la estructura de la termodinámica, consideración necesaria para establecer las interacciones multinivel que pueden establecerse para un aprendizaje significativo de la misma.

La primera ley de la termodinámica sostiene que existe una función de estado extensiva $E$, o energía total del sistema, que para cualquier proceso en un sistema cerrado se expresa así:

$$
\Delta \mathrm{E}=\mathrm{Q}+\mathrm{W}
$$

donde $\Delta E$ es la variación de la energía del sistema en el proceso $^{4}, Q$ es el calor transferido al sistema durante el proceso y $W$ es el trabajo realizado sobre el sistema en el transcurso del proceso. La variación de energía del sistema va acompañada de una variación de la energía del entorno, que se expresa como $\Delta E$, por lo que la energía total del sistema más la del medio permanece constante, esto es, se conserva, por lo que $\Delta E s i s t+$ $\Delta$ Emed $=0$.

Consecuentemente si consideramos la energía total de un cuerpo como la suma de la energía cinética $(E k)$, la energía potencial $(E p)$ y la energía interna $(U)$, tenemos:

$$
\mathrm{E}=\mathrm{Ek}+\mathrm{Ep}+\mathrm{U}
$$

Como este apartado se limita a sistemas en reposo en ausencia de campos externos, vemos que

\footnotetext{
Según Van Roon et al. (1994), un cambio del sistema desde cierto estado inicial hasta cierto estado final es llamado proceso. Un proceso acompañado por un cambio en la energía interna del sistema indica alguna clase de interacción entre el sistema y sus alrededores.
}

$E k=O=E p$, por lo que $E=U$; así, nuestra primera ecuación queda:

$$
\Delta \mathrm{U}=\mathrm{Q}+\mathrm{W}
$$

$\Delta U$ es la variación de la energía interna del sistema, $Q$ y $W$ se fijan con respecto al sistema, por lo que si del medio se transfiere energía en forma de calor al sistema, $q$ es positivo, mientras que si existe transferencia de energía del sistema al entorno en forma de calor, $q$ es negativo. Cuando el medio realiza trabajo sobre el sistema (por ejemplo, comprimiéndolo), $W$ es positivo, mientras que si el sistema realiza trabajo sobre su entorno, $W$ es negativo. Ni el calor (Q), ni el trabajo (W) son magnitudes negativas, lo que indican estos signos es su contribución a la disminución o aumento de la temperatura de un sistema. Un $Q$ y un $W$ positivos incrementaron la energía interna del sistema.

\section{Energía interna}

La energía interna (U) es una función del estado del sistema. Para cualquier proceso, $\Delta U$ sólo depende de los estados inicial y final del sistema y es independiente del camino seguido, así: $\Delta U=$ Ufinal - Uinicial. De manera más explícita, el entendimiento del concepto energía interna requiere la utilización de un modelo de partículas en el cual se describa su naturaleza corpuscular y se contribuya a su descripción cualitativa, tratamiento que consideramos debe ser previo al empleo de cualquier formalismo matemático.

Así, entendemos que las partículas son aquellas unidades reconocibles que componen un sistema en cualquier momento, cada una de las cuales tiene 
una estructura interna. Las partículas pueden constituir sistemas ${ }^{5}$ por sí solas poseen propiedades en conjunto que les confieren una determinada organización, la cual es propensa al cambio, bien sea por reacciones químicas o nucleares, o bien por una transformación de su estructura interna en procesos que involucran decaimiento radiactivo.

Para entender la dinámica de un sistema de partículas es necesario introducir el concepto de energía cinética interna del sistema, definida como la sumatoria de las energías cinéticas de las partículas. Un segundo tipo de energía, derivada de las fuerzas internas que realizan trabajo sobre las partículas, como una consecuencia de su movimiento relativo, se denomina energía de interacción interna, y se define como la sumatoria de las energías de interacción entre pares de partículas.

Un tercer tipo de energía, asociada con la estructura interna de las partículas, que se puede modificar como resultado de colisiones inelásticas y otras interacciones entre las partículas, o bien por agentes externos, es energía intrínseca de las partículas (Alonso y Finn, 1997).

Teniendo en cuenta las aclaraciones anteriores, la energía interna $(U)$ de un sistema se define como la suma de las tres energías: cinética $(E k)$, de interacción (Eint), e intrínseca de las partículas (Epart):

5 Un sistema es «un conjunto de elementos interdependientes, es decir ligados entre sí por relaciones tales que si una es modificada, las otras también lo son y que, en consecuencia, todo el conjunto es modificado», según Ludwig Von Bertalanffy (1968), fundador de la Teoría General de Sistemas.

$$
\mathrm{U}=\Sigma \mathrm{Ek}+\Sigma \text { Eint }+\Sigma \text { Epart (1) }
$$

Esta ecuación contiene todos los elementos necesarios para conocer y analizar lo que sucede en un sistema físico, por eso se llama la "ecuación para todo" (Alonso y Finn, 1997).

Vale la pena mencionar que a menudo las energías individuales de las partículas no están bien definidas, y resulta infortunado comprender la energía del sistema como la suma de dichas energías de las partículas individuales; de hecho, si consideramos que el todo, es decir, un sistema de partículas, posee una serie de propiedades que no tienen dichas partículas cuando no están separadas, conviene reconocer las energías de interacción para comprender la dinámica de dicho sistema, más allá de hablar del estado físico de las mismas, para lo cual esta pregunta resulta improcedente.

Cuando se alude a la energía intrínseca de las partículas, es necesario aclarar el tipo de sistema que se describe y la clase de comportamiento energético que manifiesta. Así, en un sistema cuyas partículas sean átomos, la energía de las partículas está asociada al movimiento electrónico.

Si hablamos de moléculas, tendríamos que describir en primer lugar la rotación de la molécula en conjunto en segundo término el movimiento relativo vibracional del núcleo y en tercera instancia el movimiento electrónico de la molécula (Levine, 1996):

Epart $=$ E (rotación) + E (vibración) + E (electrones)

Si el sistema es un núcleo, hay que describir los movimientos rotacionales y 
vibracionales colectivos y el movimiento individual de los nucleones. Para un sistema de hadrones, podemos escribir Epart $=E$ (quarks), la cual es la energía asociada con la dinámica de los quarks, que componen los hadrones (Alonso y Finn, 1997).

En algunos procesos que ocurren en estos sistemas, las partículas pueden cambiar energías intrínsecas o modificar su propia naturaleza, con la influencia de su cinética e interacciones, o bien debido a factores externos, como los choques inelásticos y las reacciones químicas y nucleares. Ésta es una prueba de lo cambiante que pueden ser las partículas y de la importancia de analizar situaciones específicas en las que participan. Es conveniente señalar que, con miras a una visión dinámica de los sistemas fisicoquímicos, pueden existir aquellos en los que se ejerce más de una interacción y otros en los que una interacción es dominante.
Como vemos, las partículas en un sistema pueden ser considerarse sistemas por sí mismas, compuestas a su vez por otras partículas. Recordemos que un postulado del pensamiento sistémico establece que existen distintos niveles de organización, lo que invita a pensar que hay sistemas dentro de sistemas, los cuales poseen propiedades emergentes que sólo pueden surgir en determinado nivel (Capra, 1996). Así, la energía de la partícula (E part) tiene energía interna cuando la partícula se trata como un nuevo sistema. Por ejemplo, los átomos pueden entenarse como partículas en un gas, pero a su vez son sistemas compuestos por electrones y un núcleo, razón de ser de la estructura atómica. A su turno, el núcleo puede considerarse un sistema compuesto de nucleones, cuyo estudio es la base de la física nuclear. Esta secuencia se puede expresar, según Alonso y Finn (1997), de la siguiente manera:

$$
\mathrm{U}=\mathrm{E}(\mathrm{K})+\mathrm{E}(\mathrm{I})+\mathrm{E}(\mathrm{P})
$$

\section{El calor y el trabajo}

¿Cómo se conserva la energía en un sistema termodinámico, de acuerdo con esta interpretación del concepto energía interna?

Los cambios en la energía interna de un sistema pueden estar asociados a los cambios en los tres términos de la ecuación (1), es decir, en intercambios de energía con otros sistemas tales como el ambiente. Por ejemplo, si tenemos un globo con helio y lo dejamos a merced de los rayos solares, cuando se incrementa la temperatura de este sistema (calentamiento), aumenta incrementa la energía cinética de las partículas [primer término en la ecuación (1)]; además, resulta un cambio en el término interacción (expansión del gas), o en el término partícula (excitación). Modificando la configuración del sistema (cambio de forma o de volumen), se produce un cambio en el término interacción, que puede afectar los otros dos términos (cambio en la temperatura del sistema o excitación electrónica). En otros sistemas, si utilizamos como 
fuentes externas de energía un horno microondas o la radiación infrarroja, cambiamos el tercer término "partícula" (absorción de radiación, por ejemplo de fotones; ionización; disociación), afectando el primer y segundo términos de la ecuación (1).

Algunos autores, como Alonso y Finn (1992, 1997), sostienen que para un mejor aprendizaje del concepto energía interna es necesario mostrar la igualdad entre el cambio de la energía interna de un sistema y la energía intercambiada con otro sistema, lo que supone tener en cuenta otro término en la ecuación general de la energía interna, la radiación (R). Por tanto, la ecuación que expresa la variación de la energía interna se escribe:

$\Delta \mathrm{U}=\mathrm{Q}+\mathrm{W}+\mathrm{R}(3)$

Donde el término $R$ es la radiación (fotones) absorbida por las partículas del sistema. La ecuación anterior, como veíamos, es la expresión de la primera ley de la termodinámica que, al tener en cuenta la radiación, también se denomina primera ley de la física.

Si relacionamos la ecuación (1) con la ecuación (3), tenemos:

$$
\Delta \mathrm{Ek}+\Delta \text { Eint }+\Delta \text { Epart }=\mathrm{Q}+\mathrm{W}+\mathrm{R}(4)
$$

Aquí se demuestra la equivalencia entre los cambios de los tres componentes de la energía interna en un sistema y los intercambios de energía con el ambiente. No es una correlación directa entre los tres términos de la derecha y los tres de la izquierda, pero $Q$ se asocia con el cambio en la energía cinética de las partículas, generalmente las colisiones entre las partículas del sistema y las del entorno, como veremos más adelante; el trabajo se asocia con un cambio en el valor del término interacción, que va desde el cambio de forma y volumen del sistema y la orientación y configuración de las partículas; $R$ se asocia con un cambio en el término energía de la partícula, por absorción o emisión de fotones, la cual puede afectar a los otros dos términos en el caso de los rayos microondas.

Como vemos, un acercamiento a nuestra propuesta de entender la energía interna, el calor, el trabajo y la temperatura desde la utilización de un modelo de partículas confirma el propósito de considerarlos parte de una visión dinámica del mundo, en la que el lenguaje cobra sentido, por lo que una perspectiva integrada de la energía proporciona elementos de juicio para la profundización en un campo conceptual que permite la unificación de las teorías fisicoquímicas, otrora ideal científico en los siglos XIX y XX.

Como sabemos, los sistemas termodinámicos están formados por un número enorme de partículas, una cifra de referencia denominada número de Avogadro, la cual nos demuestra que las propiedades termodinámicas deben estudiarse como valores promedio de conjuntos estadísticamente grandes de partículas. De la ecuación que expresa la energía total de un sistema, deducimos su valor constante, siguiendo las conclusiones de la primera ley de la termodinámica. Pero ¿qué ocurre en el campo atómico-molecular? Para contestar esta pregunta debemos reconocer un aspecto nuevo del modelo, en el que la interacción y el comportamiento de las partículas queden en evidencia. 
En el caso de la energía cinética de las partículas, inferimos que si todas se mueven en la misma dirección, con la misma velocidad y, si se permite el término, al unísono, el sistema se comporta como una partícula individual; este es el caso de un objeto que se desplaza sobre una superficie que ejerce la mínima fricción, donde la energía cinética de todas sus partículas aumenta, lo que comprueba que se comunica energía al objeto. De igual manera, si elevamos el objeto desde el suelo, se mueven todos sus átomos de modo coherente, donde se transfiere una energía que se almacena en forma de energía potencial en todos sus átomos. Ambas situaciones ejemplifican la transferencia de energía cuando realizan un trabajo sobre el sistema, en el que se comunica a sus partículas un movimiento coherente, lo que también ocurre cuando el sistema hace un trabajo sobre su entorno (Atkins, 2000).

Si aplicamos al objeto una llama, las direcciones y las velocidades de las partículas no tendrían correlación, por lo que el movimiento se describe como incoherente, ya que la energía se almacena en forma de movimiento térmico de los átomos. Así, cuando se calienta un sistema, se transmite a las partículas un movimiento incoherente, por lo que se define el calor como un proceso en el que se transfiere energía de un sistema a otro por medio del movimiento incoherente de sus partículas. De acuerdo con los paradigmas vigentes en termodinámica, compartimos los planteamientos de Levine (1996), Atkins (1992, 2000) y Alonso-Finn $(1992,1997)$ en relación con el trabajo y el calor, quienes consideran que el calor y el trabajo sólo pueden definirse en términos de procesos, por lo que resulta improcedente hablar de calor y trabajo, antes y después del proceso de transferencia de energía. El calor es la transferencia de energía por medio del movimiento incoherente de las partículas, debido a una diferencia de temperatura. El trabajo es una transferencia de energía mediante el movimiento coherente de las partículas, stet a una fuerza macroscópica que actúa a lo largo de una distancia.

\section{La temperatura}

Según Levine (1996), la temperatura es una propiedad común a los sistemas en equilibrio térmico, hecho que se expresa de la siguiente manera y constituye el fundamento de la ley cero de la termodinámica, denominada así porque una vez establecidas la primera, segunda y tercera leyes, se advirtió su importancia en el desarrollo de la ciencia:

\section{"Dos sistemas en equilibrio térmico con un tercero lo están entre sí"}

Este enunciado permite reconocer la existencia de la temperatura como una función de estado, que produce cambios observables en las propiedades macroscópicas de la materia. La ecuación de estado de un gas ideal relaciona las propiedades macroscópicas, presión $(P)$, volumen $(V)$ y temperatura $(T)$.

Al estudiar un sistema de muchas partículas y considerar el comportamiento promedio de sus componentes, la presión ejercida por el sistema de partículas en términos de los choques que experimentan las moléculas del gas contra las paredes del recipiente es un valor que, entre otros, sirve para concluir que la temperatura se constituye en la 
medida de la energía cinética promedio de las partículas de un sistema.

A manera de conclusión, el objeto de conocimiento de la termodinámica se circunscribe en el estudio de las interacciones entre la materia y la energía, teniendo como puntos de referencia la estructura ${ }^{6}$ y la organización de los sistemas materiales a partir del análisis de referentes que involucran la naturaleza corpuscular. Así, la configuración de la termodinámica como un campo prolífico en el que se procura enriquecer aquella visión de mundo con la cual se describen e interpretan los fenómenos se robustece con la valoración de los problemas, los experimentos, el formalismo matemático y la evolución de los conocimientos, para lo cual se priorizan conceptos que hacen parte de su base empírica, tales como la energía interna, el calor, la temperatura y el trabajo. Tal conceptualización supone una progresión hacia formas más complejas de analizar los hechos y los fenómenos, logrando construir así un esquema de conservación, transformación, transferencia y degradación de la energía.

\section{Aspectos pedagógicos y didácticos}

La educación en ciencias promueve, desde una perspectiva compleja, la reconstrucción de los procesos culturales de comunicación, en lo que al fortalecimiento de la cultura escolar se refiere, al asumirse la dialéctica y el diálogo entre lógicas antagónicas (dialogicidad) como formas de interactuar y dar sentido a la

6 La estructura se concibe como"un sistema de transformaciones, que implica leyes como sistema y que se conserva o enriquece por el juego de transformaciones, y comprende totalidad, transformaciones y autorregulaciones" (Ramírez, 2003). realidad. En este sentido, el objetivo de la enseñanza de las ciencias de procurar un cambio conceptual ha sido replanteado para abordar también aspectos metodológicos, actitudinales y sociológicos.

Sin un enfoque metodológico las ideas de los estudiantes se encuentran descontextualizadas, pues un cambio conceptual exclusivo podría asociarse con un simple cambio del contenido de las concepciones, recalcándose una vez más los conocimientos declarativos en contra del aprendizaje significativo. Por esta razón se hace prioritario aproximar a los estudiantes a las características del trabajo científico, como la interpretación de situaciones problemáticas, la emisión de hipótesis, el diseño experimental, el análisis de resultados, su coherencia global y demás actividades propias de un proceso de aprendizaje de las ciencias como investigación orientada, donde los estudiantes se consideran "investigadores noveles", que trabajan en equipos cooperativos realizando investigaciones, donde el profesor es un experto conocedor de las mismas.

Gil y colaboradores (Gil, 1993; Gil, 1994; Furió y Guisasola, 2001) proponen unas estrategias flexibles de trabajo enmarcadas en el aprendizaje de las ciencias como investigación orientada, las cuales permiten una interpretación multidimensional de la dinámica escolar:

1. Se plantean situaciones problemáticas que motiven al estudiante y que proporcionen una concepción preliminar de la tarea.

2. Se propone un estudio cualitativo de las situaciones problemáticas y la toma de decisiones, utilizando búsquedas bibliográficas, con el fin 
de que los estudiantes delimiten el problema y expliciten sus ideas.

3. Los problemas se trabajan siguiendo una orientación científica con el objeto de que se emitan hipótesis (explicitándose las ideas previas), se elaboren estrategias de resolución y se cotejen los resultados con los de otros grupos, espacio propicio para los conflictos cognoscitivos y el análisis de nuevas hipótesis.

4. Los nuevos conocimientos se aplican a nuevas situaciones para afianzarlos a medida que se profundiza en éstas, enfatizando en las relaciones ciencia, tecnología, sociedad y ambiente (CTSA).

5. En todo el proceso es prioritario favorecer las actividades de síntesis, la elaboración de productos como mapas conceptuales, memorias, etc., y la concepción de nuevos problemas.

El objetivo de mejorar la forma como el estudiante construye conocimientos y comprende la realidad se puede analizar a partir del tratamiento de situaciones problemáticas motivantes -como afirma Bachelard (1932) dentro del psicoanálisis del interés-, las cuales son afines con una propuesta de aprendizaje por investigación. Es oportuno enunciar que dentro de las estrategias metodológicas que evidencian un tratamiento científico de los problemas está la implementación de programas-guía de actividades (Gil, 1988), propuesta que se basa en la aplicación del modelo investigativo en la elaboración de secuencias de actividades, las cuales propician un manejo metacognitivo del aprendizaje de las ciencias. En consecuencia, valdría la pena preguntarnos qué tipo de propuesta metodológica y didáctica permite la construcción y la reconstrucción de significados, formas de ser, significar y actuar.

Si analizamos un campo específico del saber físicoquímico como el termodinámico, podemos aplicar una estrategia didáctica para propiciar un aprendizaje significativo de esta temática. Un supuesto básico en la puesta a prueba del modelo de resolución de problemas como investigación propone el acercamiento de los estudiantes a las características del trabajo científico (Gil, 1991), las cuales se centran en el principio de poner a los alumnos en situación de plantear problemas que hay que investigar, explicitando un modelo didáctico que permita la evolución del pensamiento, al igual que interpretar el tipo de interacciones discursivas que esbozan los individuos involucrados en este proceso (Dumrauf y Cordero, 2004).

\section{El modelo didáctico de enseñanza- aprendizaje por investigación en el aprendizaje de los conceptos energía interna, calor y temperatura}

Entre las características de un modelo didáctico subyacen, por una parte, aspectos relacionados con la dimensión explicativa de los acontecimientos que, en suma, hacen referencia a la concepción o imagen de los procesos educativos y, por otra, la dimensión normativa relacionada con los esquemas que indican cómo orientar, dirigir y actuar en la práctica educativa. En términos generales, el modelo didáctico encauzado en una filosofía de la actuación debe configurarse en una práctica educativa cargada de una ideología determinada, que permita a su vez consolidar un tipo de sociedad dinámica, 
cambiante, con incertidumbres propias de un sistema organizado.

En relación con este eje direccionador del currículo, la investigación como principio didáctico se caracteriza por favorecer una visión evolucionista de los conocimientos, al igual que permite reflexionar en torno a las ideas que posee el estudiante, en un clima de autonomía, propio de discusiones con un fuerte carácter académico. El profesor no sólo se reduce a dirigir las actividades, sino que consolida su liderazgo como experto en el análisis temático y, más aún, como evaluador del proceso, en el que se censura el alcance de los programas guías de actividades y se fomenta un despliegue de las competencias de los estudiantes.

Los ejes sobre los cuales se construye la actividad investigativa en el aula a partir del modelo de resolución de problemas incluyen los siguientes aspectos (Gil, 1993):

\section{Considerar cuál puede ser el interés de la actividad problemática abordada} "El amor por la ciencia debe ser un dinamismo psíquico autógeno. En el estado de pureza logrado por un psicoanálisis del conocimiento, la ciencia es la estética de la inteligencia". Bachelard (1934).

Dicho interés proporciona el desarrollo de actitudes científicas, hacia la ciencia y su aprendizaje, así como un estudio de las estrechas relaciones entre la ciencia, la tecnología, la sociedad y el ambiente. Se intenta que sean los mismos estudiantes quienes planteen el problema, a partir del examen de una situación abierta que ponga a prueba su ingenio y brinde posibilidades de actuación al asumir esta actividad como un trabajo de investigación.

\section{Comenzar por un estudio cualitativo} de la situación, intentando acotar y definir de manera precisa el problema, explicitando las condiciones reinantes "Una experiencia que no rectifica ningún error, que es meramente verdadera, que no provoca debates, ¿para qué sirve?". Bachelard (1934).

Sin conocimientos nadie puede aventurarse a evaluar caminos de actuación para la resolución de problemas; por eso deben considerarse fundamentales la identificación y el abordaje de cuerpos conceptuales que permitan el estudio riguroso del objeto de conocimiento científico, con lo cual se evitan las afirmaciones difusas, producto de un análisis enmarcado dentro de la metodología de la superficialidad.

Por tanto, todo modelo de aprendizaje por investigación que se desarrolle en el aula debe estar soportado por un programa de investigación que permita, con un cuerpo de conocimientos y una metodología coherente con los mismos, facilitar el despliegue del componente imaginativo e innovador para la toma de decisiones en torno a la delimitación de la situación y la explicación de lo que se intenta determinar.

\section{Emitir hipótesis fundadas sobre los factores de los que puede depender la magnitud buscada y sobre la forma de esta dependencia, imaginando, en particular, casos límite de fácil interpretación física}

"Se ha dicho frecuentemente que una hipótesis científica que no levanta ninguna contradicción no está lejos de ser una hipótesis inútil”. Bachelard (1934).

La importancia de la formulación de hipótesis radica en el hecho de rechazar la idea de un conocimiento cierto, 
acabado, por un conocimiento creativo, por cuanto deja de lado lo evidente y se concentra en lo divergente, que proporciona a su vez una consideración más rigurosa del razonamiento, sometiendo a prueba los resultados.

4. Elaborar y explicitar posibles estrategias de resolución antes de proceder a ésta, evitando el puro ensayo y error. Buscar distintas vías de resolución para posibilitar la contrastación de los resultados obtenidos y mostrar la coherencia del cuerpo de conocimientos de que se dispone

"El espíritu científico nos impide tener opinión sobre cuestiones que no comprendemos, sobre cuestiones que no sabemos formular claramente. Ante todo es necesario saber plantear los problemas. $Y$ dígase lo que se quiera, en la vida científica los problemas no se plantean por sí mismos. Es precisamente este sentido del problema el que caracteriza el verdadero espíritu científico. Para un espíritu científico, todo conocimiento es una respuesta a una pregunta. Si no hubo pregunta, no puede haber conocimiento científico. Nada es espontáneo. Nada está dado. Todo se construye”. Bachelard (1934).

La importancia del cuerpo de conocimientos radica en su papel clave dentro de la representación del problema, la modelización de la situación, la emisión de hipótesis y la búsqueda de vías de resolución. Pero estas estrategias de resolución, que se constituyen como los diseños experimentales, son también opcionales en la medida en que requieren imaginación y ensayos, pues no se obtienen directamente del cuerpo teórico sino que se asumen como una tarea abierta, de consensos.
5. Realizar la resolución verbalizando al máximo, fundamentando lo que se hace y evitando, una vez más, operativismos carentes de significación

"Los profesores de ciencias no han reflexionado sobre el hecho de que el adolescente llega al curso de física con conocimientos empíricos ya constituidos; no se trata, pues, de adquirir una cultura experimental, sino de cambiar una cultura experimental, de derribar los obstáculos amontonados por la vida cotidiana". Bachelard (1934).

La planificación de la estrategia de resolución implica el evitar recaer en actividades de ensayo y error, para constituir una verbalización alejada de los procesos operativos, manifestando así los principios aplicados para la elaboración y puesta en práctica de estrategias de resolución, incluyendo, en su caso, el diseño y realización de montajes experimentales para someter a prueba las hipótesis a la luz del cuerpo de conocimientos de que se dispone. Son claves en el proceso el análisis y la comunicación de los resultados, cotejándolos con los obtenidos por otros grupos de estudiantes y por la comunidad científica.

\section{Analizar cuidadosamente los} resultados a la luz de las hipótesis elaboradas y, en particular, de los casos límite considerados

"Queda luego la tarea más difícil: poner la cultura científica en estado de movilización permanente, remplazar el saber cerrado y estático por un saber abierto y dinámico, dialectizar todas las variables experimentales, dar finalmente a la razón motivos para evolucionar". Bachelard (1934). 
En la contrastación de los resultados con base en las hipótesis y el cuerpo de conocimiento, resulta enriquecedor evaluar este proceso, con lo que Reiff (citado por Ramírez, 1990) considera la verificación de la consistencia interna:

1. ¿Es razonable el valor de la respuesta?

2. ¿Depende la respuesta de una forma cualitativa de los parámetros del problema, en el sentido que cabría esperar?

3. ¿Se ajusta la respuesta a lo que se podría esperar en situaciones sencillas y especiales (por ejemplo, las correspondientes a valores extremos de las variables)?

4. ¿Se obtiene la misma respuesta por otro medio diferente de resolución?

Los resultados pueden dar origen a nuevas investigaciones, a nuevos problemas, ampliando la gama de actividades interesantes para el estudiante, convirtiéndose en ocasión para destacar las relaciones ciencia, tecnología, sociedad y ambiente (CTSA) que enmarcan el desarrollo científico.

La idea es comunicar al estudiante lo importante que resulta construir conocimiento científico como una actividad compleja, colectiva y motivante, para lo cual el programa de actividades se convierte en un programa de investigación $y$, a su vez, en una propuesta curricular en la que el desarrollo de competencias científicas tenga un papel prioritario.

De hecho, el modelo de aprendizaje por investigación supone en primer lugar crear un espacio de reflexión en torno a los programas-guía de actividades, es decir, situaciones problemáticas susceptibles de implicar a los estudiantes en una investigación dirigida. Así
Driver y Oldham (citados por Medina, 1996) sostienen:

Quizá la más importante implicación del modelo constructivista es concebir el currículo no como un conjunto de conocimientos y habilidades, sino como el programa de actividades a través de las cuales dichos conocimientos y habilidades se puedan construir.

El valor de esta propuesta radica en el manejo investigativo de los acontecimientos científicos, que a su turno posibilitan la construcción de conocimientos a partir de una secuencia de enseñanza cuidadosamente elaborada, pero flexible a cambios y modificaciones que resulten de su implementación. Las actividades que forman parte de los programas-guía pueden ser muy diversas, pero se centran en tres aspectos propios de la dinámica del aula de clase (Erazo y Tiusaba, 1995): actividades de iniciación (sensibilización hacia el tema, explicitación de las ideas que posean los estudiantes, etc.), actividades de desarrollo (introducción de conceptos científicos, manejo reiterado de dichos conceptos, detección de errores, emisión y fundamentación de hipótesis, conexión entre partes distintas de la asignatura, elaboración de diseños experimentales, etc.) y actividades de finalización (elaboración de síntesis, esquemas, mapas conceptuales, V-epistemológicas, redes conceptuales, mentefactos, etc.).

El tipo de tratamiento que se realiza en torno al programa-guía consiste en ejecutar cada una de las actividades trabajando en pequeños grupos, con el fin de propender por la consolidación de un espacio para la discusión, la participación y la motivación, a sabiendas que la construcción de conocimientos 
científicos se hace en una comunidad académica. Así, la labor del docente consiste en supervisar el trabajo de los estudiantes, realizar un seguimiento de las tareas abordadas y coordinar la puesta en común reformulando los resultados, al mismo tiempo que aclara y complementa la labor de los grupos.

En el caso particular de la termodinámica, la elaboración del programa de investigación, en el que se referencian los obstáculos epistemológicos dentro de la construcción de los conceptos energía interna, calor y temperatura, sigue la evolución de los conocimientos termodinámicos, convirtiéndose en una perspectiva válida para evaluar los grandes problemas y dificultades históricas que van desde la aparición del concepto vis viva, el reconocimiento del calórico como una hipótesis propia de la "metodología de la superficialidad”, hasta las explicaciones atómico-moleculares que dan cuenta de la interacción entre sistemas de partículas.

Algunos de los indicadores de comprensión conceptual de la energía, el calor y el trabajo, en relación con la secuenciación de los temas de física, pueden enmarcarse en la propuesta de Verdú (2005):

1. Disponer de un concepto cualitativo adecuado de energía, lo que supone conocer que las transformaciones que experimenta un sistema se deben a las interacciones con otros sistemas o a las interacciones entre sus partes, es decir, a la capacidad de la materia para interaccionar de diversas maneras.
2. Significado físico de los conceptos trabajo y calor y su relación con la energía. Las variaciones de energía de un sistema pueden deberse a la realización de trabajo o calor.

3. Conservación, transformación y degradación de la energía.

Teniendo en cuenta estos planteamientos, a continuación se ejemplifica un problema que forma parte de un programa-guía de actividades enfocado al aprendizaje significativo de los conceptos calor, temperatura y energía interna.

\section{Implementación del modelo de partículas (encendido / apagado)}

\section{Problema}

Dos cuerpos, $A$ y $B$, entran en contacto a diferente temperatura. $B$ es el doble que A. ¿Cómo podría describir la temperatura final de los cuerpos, utilizando para ello un modelo teórico en el que usted se represente como un observador molecular?

Con base en la discusión anterior, una estrategia para retroalimentar el trabajo en torno a los conceptos energía, calor y temperatura se centra en la utilización de un modelo de transferencia de energía presentado por Atkins (1984) y Ben Zvi et al. (1993), el cual representa la interacción de dos objetos que satisfacen las condiciones del problema planteado a los estudiantes. En este modelo se describe el movimiento coherente e incoherente de las partículas en función del trabajo y el calor. 

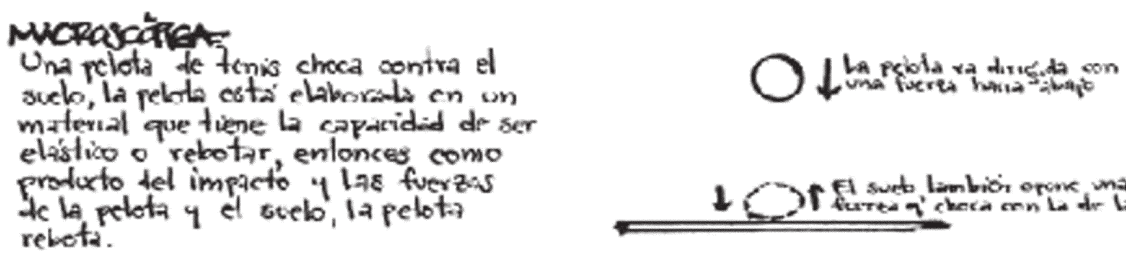

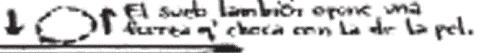

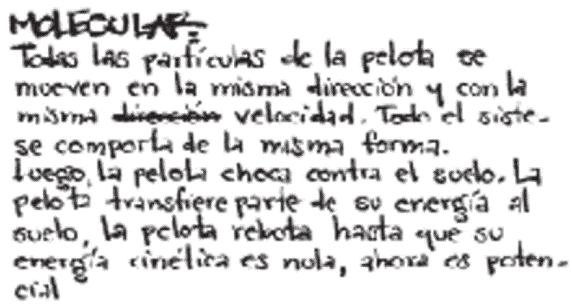

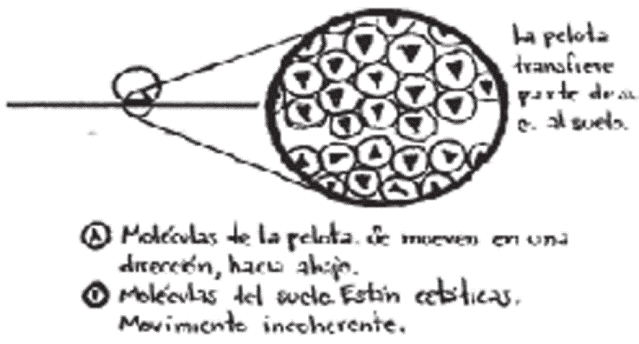

Gráfico 1. Descripción del movimiento coherente e incoherente por parte de un estudiante.

Para el problema en cuestión se estableció como consenso general que, en la modelización del objeto, A tuviese tres partículas y tres unidades de energía, mientras que el objeto B tendría seis partículas con energía muy baja cercana a cero.

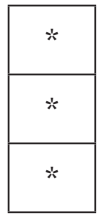

A

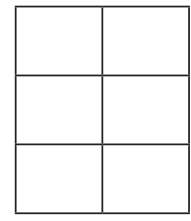

B
Figura 4. Representación de dos sistemas que entran en contacto. A tiene tres unidades de energía y $B$ tiene un valor de energía bajo.

Cuando los dos cuerpos se ponen en contacto, las unidades de energía pueden moverse desde una partícula encendida (marcada con asterisco) en el objeto A, hasta las partículas apa- gadas del objeto $\mathrm{B}$. Al plantearse que el número de formas posibles como el sistema, integrado por los dos objetos, puede conservar su energía, se llega a la conclusión por parte de los estudiantes (más del 80\%) de que las propiedades termodinámicas de un sistema son valores promedio de conjuntos estadísticamente enormes de partículas. Al completar la tabla en la que se registra el número de formas en las cuales el sistema puede mantener su energía, se deduce que los estudiantes utilizaron estrategias diversas para analizar las probabilidades de transferencia energética. Uno de estos procedimientos consistió en la elaboración de tableros y fichas para manipular todas las posibilidades.

Como ejemplo de probabilidades se muestran seis de estas formas, donde se transfiere una unidad de energía es transferida del objeto A al B. En total, si una de las tres unidades de energía del objeto A es transferida al objeto B, el 
sistema puede mantener su energía de 18 formas posibles. Sin embargo, existe una sola manera en la cual el sistema puede mantener su energía si toda se concentra en el objeto A, y 20 posibles formas si toda la energía se transfiere al objeto B.

Como es de recalcar, el 100\% de los estudiantes de la muestra (maestros en formación de licenciatura en biología), con la orientación del docente, llegan a la conclusión de que la relación entre el número de partículas prendidas y el número de partículas apagadas puede ser un indicador de la temperatura del sistema (Porras, 2005). Como vemos en la tabla 1, más de la mitad de las formas en que se puede describir la transferencia energética de un sistema a otro obedece a la igualdad de sus temperaturas. Estos cálculos del número de formas en que el sistema puede mantener su energía se condensan en la siguiente tabla:

Tabla 1. Número de formas en que se puede transferir la energía del objeto $A$ al $B$.

\begin{tabular}{|c|c|c|c|c|c|c|}
\hline & \multicolumn{2}{|c|}{ Unidades de energía } & \multirow{2}{*}{\multicolumn{2}{|c|}{$\begin{array}{c}\text { Partículas prendidas } \\
\text { Relación ----artículas apagadas }\end{array}$}} & \multirow[t]{3}{*}{$\mathrm{N}^{\circ}$ de formas } & \multirow{3}{*}{$\begin{array}{c}\mathrm{N}^{\circ} \text { de formas } \\
(\%)\end{array}$} \\
\hline & & & & & & \\
\hline & Objeto A & objeto B & Objeto A & objeto B & & \\
\hline I & 3 & 0 & $3 / 0$ & $0 / 6$ & 1 & 1,2 \\
\hline II & 2 & 1 & $2 / 1$ & $1 / 5$ & 18 & 21,4 \\
\hline III & 1 & 2 & $1 / 2$ & $1 / 2$ & 45 & 53,6 \\
\hline IV & 0 & 3 & $0 / 3$ & 1 & 20 & 23,8 \\
\hline
\end{tabular}

El análisis de la tabla nos sugiere dinamizar la complejidad de situaciones del aula hacia conclusiones, admitidas en el consenso de la clase, tales como:

1 Si dos objetos se ponen en contacto a diferentes temperaturas, éstas se igualarán, incluyendo el entorno en el que se encuentren.

2 La temperatura de un objeto no está relacionada con su tamaño.

3 Si dos objetos a la misma temperatura se ponen en contacto, la elevación de la temperatura que experimenta uno de ellos es despreciable.
4 Una situación termodinámica existe dentro de una conducta relacional propia del lenguaje, catalogándose como la más probable ya que describe el máximo número de formas. En este sentido, la conceptualización de la temperatura aceptada como consenso de la clase es:

La temperatura es una función de estado y se constituye en una magnitud intensiva, que en términos atómico-moleculares se describe como la energía cinética promedio de las partículas que componen un sistema. $\Delta$ 


\section{Bibliografía}

Alonso, M. y Finn, E. (1992). Física. Vol. 1. Wilmington, Delaware: Adisson-Wesley Iberoamericana.

Alonso, M. y Finn, E. (1995). An integrated approach to thermodynamics in the introductory physics course. Physics Teacher. 33.

Alonso, M. y Finn, E. (1997). On the notion of internal energy. Physics Education, 32 (4), 256-264.

Atkins, P. W. (1991). Fisicoquímica. Wilmington, Delaware: Adisson-Wesley Iberoamericana.

Atkins, P. W. (1992). The Second Law. Estados Unidos: Scientific American Editor.

Atkins, P. W. and Jones, L. (2000). Chemical principles: The quest for insight. Nueva York: W. H. Freeman and Company Editors.

Atkins, P. W. (2003). El dedo de Galileo: diez grandes ideas de la cencia. España: Editorial Espasa Calpe..

Bacon, F. (1980). Novum organum. México: Porrúa.

Bachelard, G. (1982). La formación del espíritu científico. México: Siglo XXI Editores.

Bartell, L. (2001). Stories to Make Thermodynamics and Related Subjects More Palatable. Journal of Chemical Education, 78 (8), 1059-1067.

Bensaude-Vincent, B. y Stengers, I. (1997). Historia de la química. Madrid: Addison-Wesley Iberoamericana.

Bertalanffy, L. V. (1968). Teoría general de los sistemas: fundamentos, desarrollo, aplicaciones. Bogotá: Fondo de Cultura Económica.

Black, J. (1803). Lectures on the elements of chemistry delivered in the Univer- sity of Edinburgh. http: //webserver. lemoyne.edu/faculty/GIUNTA (febrero de 2006).

Campanario, J. y Moya, A. (1999). ¿Cómo enseñar ciencias? Principales tendencias y propuestas. Enseñanza de las Ciencias. 17 (2), 179-192.

Capra, F. (1996). La trama de la vida. Una nueva perspectiva de los seres vivos. Barcelona: Editorial Anagrama.

Cárdenas, F. (1998). Desarrollo y evaluación de los procesos de razonamiento complejo en ciencias. Ciencia y Tecnología. 3, 53-68.

Carrascosa, A y Gil, D. (1985). La "metodología de la superficialitat" il'aprenentatge de les ciéncies. Enseñanza de las Ciencias, 113-120.

Domínguez Castiñeiras, J. M, et al. (1998). Las partículas de la materia y su utilización en el campo conceptual de calor y temperatura: un estudio transversal. Enseñanza de las Ciencias, 16 (3), 461-475.

Dumrauf, A. y Cordero, S. (2004). ¿Qué cosa es el calor? Interacciones discursivas en una clase de física. Revista Electrónica de Enseñanza de las Ciencias, 3 (2).

Erazo, M. y Tiusaba, E. (1995). Hacia una enseñanza de las ciencias por investigación. Educación y Cultura, 38.

Gil, D. (1983). Tres paradigmas básicos en la enseñanza de las ciencias. Enseñanza de las Ciencias, 26-33.

Gil, D. (1993). Contribución de la historia y de la filosofía de las ciencias al desarrollo de un modelo de enseñanza / aprendizaje por Investigación. Enseñanza de las Ciencias, 11(2), 197-212. 
Gil, D. (1999). El modelo constructivista de enseñanza / aprendizaje de las ciencias: una corriente innovadora fundamentada en la investigación. Organización de Estados Iberoamericanos. http: //oei.org.co/oeivirt/gilo2. htm.

Gil Pérez, D. y Martínez-Torregrosa, J. (1987). Los programas-guía de actividades: una concreción del modelo constructivista de aprendizaje de las ciencias. Investigación en la Escuela, 3, 3-12.

Holton, G. (1986). Introducción a los conceptos y teorías en las ciencias físicas. Madrid: Reverte.

Joule, J. P. (1845). On the existence of an equivalent relation between heat and the ordinary forms of mechanical power, Philosophical Magazine, 27, 205. http: //webserver.lemoyne.edu/ faculty/GIUNTA (febrero de 2006).

Kuhn, T. S. (1992). La estructura de las revoluciones científicas. México: Fondo de Cultura Económica.

Lang da Silveira, F. y Moreira, M. A. (1996). Validación de un test para verificar si el alumno posee concepciones científicas sobre calor, temperatura y energía interna. Enseñanza de las Ciencias, 14 (1), 75-86.

Levine, I. N. (1996). Fisicoquímica. Madrid: McGraw-Hill.

Medina, J. N. (1994). Evolución de las concepciones sobre las ciencias naturales, su enseñanza y su aprendizaje. Concreción y experimentación del modelo didáctico de enseñanza y aprendizaje por investigación. Tesis de maestría en docencia de la química. Bogotá: Universidad Pedagógica Nacional.

Michinel, J. L, y D’Alessandro, A. (1994). El concepto de energía en los libros de texto: de las concepciones previas a la propuesta de un nuevo sublenguaje. Enseñanza de las Ciencias, 12 (3), 369-380.

Mora, W. (1992). Las actitudes de los estudiantes hacia la imagen de las ciencias. Una estrategia metodológica para su Mejoramiento. Tesis de Maestría. Bogotá: Universidad Pedagógica Nacional.

Moreira, M. A. (2003). Lenguaje y aprendizaje significativo. Conferencia de cierre del IV Encuentro Internacional sobre Aprendizaje Significativo. Maragogi, Brasil.

Novak, J. D. (1988). Constructivismo humano: un consenso emergente. Enseñanza de las ciencias, 6 (3), 213223.

Novak, J. D. y Gowin, D. (1988). Aprendiendo a aprender. Barcelona: Ediciones Martínez Roca.

Popper, K. (1962). La lógica de la investigación científica. Madrid: Tecnos.

Porlán, R. (1990). Hacia una fundamentación epistemológica de la enseñanza. Investigación en la Escuela, 10, 3-21.

Porras, Y. (2005). El aprendizaje significativo de los conceptos calor, temperatura y energía interna, una concreción de la propuesta de aprendizaje de la química como investigación orientada. Tesis de maestría en docencia de la química. Bogotá: Universidad Pedagógica Nacional.

Pozo, J. I. y Gómez Crespo, M. A. (1998). Aprender y enseñar ciencias. Del conocimiento cotidiano al conocimiento científico. Madrid: Morata.

Ramírez, A. 2003. Área de física. Programa de Formación Permanente de Profesores de Educación Básica y Media. Enseñanza- Aprendizaje de las Ciencias por Investigación. Bogotá: Universidad Pedagógica Nacional. 
Ramírez Castro, J. L. (1990). La resolución de problemas de física y de química como investigación en la enseñanza media: un instrumento de cambio metodológico. Tesis de doctorado. Universidad Autónoma de Barcelona.

Salcedo Torres L. E, y Villarreal, M. (1999). Concepciones y acciones de los profesores de química sobre la evaluación. Revista de Educación y Pedagogía. XI, 175-207.

Thomson, W (Baron Kelvin). (1852). On the dynamical theory of heat, with numerical results deduced from $\mathrm{Mr}$ Joule's equivalent of a thermal unit, and M. Regnault's Observations on Steam. Phil. Ma,. 4, 174. http: //webserver.lemoyne.edu/faculty/GIUNTA (febrero de 2006).
Thompson, B. (1798). Heat is a form of motion: An experiment in Boring Cannon. Philosophical Transactions, 88. http: //webserver.lemoyne.edu/faculty/ GIUNTA (febrero de 2006).

Varela, M. P. y Martínez, M. M. (1997). Una estrategia de cambio conceptual en la enseñanza de la física: la resolución de problemas como actividad de investigación. Enseñanza de las Ciencias, 15 (2), 173-188.

Vásquez Díaz, J. (1987). Algunos aspectos a considerar en la didáctica del calor. Enseñanza de las ciencias, 5 (3), 235-238.

Van Roon, P. H.; Van Sprang, H. F . y Verdonk, A. H. (1994). "Work" and "Heat": on a road towards thermodynamics. International Journal of Science Education, 16(2), 131-144. 